\title{
SAS wave experiment on board Magion 4
}

\author{
J. Błęcki ${ }^{1}$, S. Gadomski ${ }^{1}$, J. Juchniewicz ${ }^{1}$, V. Korepanov ${ }^{4}$, Z. Krawczyk ${ }^{1}$, S. Savin ${ }^{3}$, \\ J. Słomiński ${ }^{1}$, P. Triska ${ }^{2}$, J. Vojta ${ }^{2}$, R. Wronowski ${ }^{1}$ \\ ${ }^{1}$ Space Research Centre PAS, Bartycka 18A ,00716, Warsaw, Poland \\ 2 Institute of Physics of Atmosphere ASCR, Bocni II, 1401-141 31 Praha 4, Czech Republic \\ ${ }^{3}$ Space Research Institute RAS, 117810, Profsoyuznaya 84/32, GSP-7, Moscow, Russia \\ ${ }^{4}$ Special Design Division of National Academy of Sciences of Ukraine, 5A Naukova str. 29060 Lvov, Ukraine
}

Received: 12 June 1996 / Revised: 11 December 1996 / Accepted: 13 January 1997

\begin{abstract}
A short description of the SAS (subsatellite analyser of spectra) wave experiment on board the Magion-4 subsatellite is given. We present first measurements of the magnetic-field fluctuations in the frequency range $32-2000 \mathrm{~Hz}$ obtained in the magnetotail during the disturbed period at the magnetopause and in the polar cusp.
\end{abstract}

\section{Introduction}

Plasma waves in the magnetosphere have been measured for more than 30 years (Shawhan, 1979). Our current knowledge of the processes in the space plasma allows us to say that waves give basic information about conditions in the different regions of the magnetosphere.

Examination of the plasma wave in the space plasma is important for the several following reasons. In collisionless plasmas waves are the source of the anomalous dissipation and play a crucial role in the energy, mass and momentum transport across the boundaries. They are useful indicators for identification of events and structures. Waves provide timing of events limited, in principle, to the inverse of the frequency of the wave being studied. This is usually of a higher time resolution than is available to other instrumental techniques. Space plasma gives the unique possibility to realise very pure experimental conditions to study basic processes. Waves give information on the microphysics of the processes in plasmas.

The goal of the plasma-wave SAS (spectrum analyser for subsatellite) experiment on board of the Magion-4 subsatellite is to work together with the wave experiment ASPI on the main satellite Interball Tail Probe. The idea of performing the same or very similar experiments on two or more objects is associated with the necessity to separate the spatial and temporal variations of the measured parameters. If the waves are measured at one point we can determine the spectrum of the measured parameter in the frequency space. In many cases, knowledge of the spectrum in the wave numbers space is required. This information can be evaluated from the correlative multipoint studies of the wave parameters.

\section{Description of the experiment}

The wave experiment ASPI on the main satellite is described in Klimov et al. $(1995,1997)$ (the latter is also in this issue), where more detailed discussion about the scientific goals of wave measurements in the different regions of the magnetosphere is presented; because our experiment is complementary to ASPI, we will not repeat this discussion. Instead we concentrate on the description of the wave experiment on board Magion 4 with SAS as the central unit. The spectrum analyser SAS can perform analysis in two parallel channels in the ULF and VLF ranges. One channel is permanently connected to the electric field sensors and can analyse the electric field signal in the frequency range $0.1 \mathrm{~Hz}-$ $400 \mathrm{kHz}$. The second channel can analyse one signal chosen from among the following: the magnetic component in the frequency range $400 \mathrm{~Hz}-20 \mathrm{kHz}$; one of three components $B_{x}, B_{y}, B_{z}$ (selected through the multiplexer) $32 \mathrm{~Hz}-2 \mathrm{kHz}$; the electric field in the frequency range $32-2000 \mathrm{~Hz}$, and one of two components of the current density.

Unfortunately, the electric field sensors and current probes do not give signals with high-enough value over the noise level. Only the measurements of the magneticfield spectra in the frequency range $32-2000 \mathrm{~Hz}$ are satisfactory. Figure 1 shows the block diagram of the SAS wave experiment.

\subsection{Magnetic-field measurements}

The three components $B_{x}, B_{y}, B_{z}$ of the magnetic field are measured by the search-coil sensors in the frequency range $1 \mathrm{~Hz}-2 \mathrm{kHz}$. The $B_{z}$-component is parallel to the rotation axis oriented to the Sun; while $B_{x}$ and $B_{y}$ are 


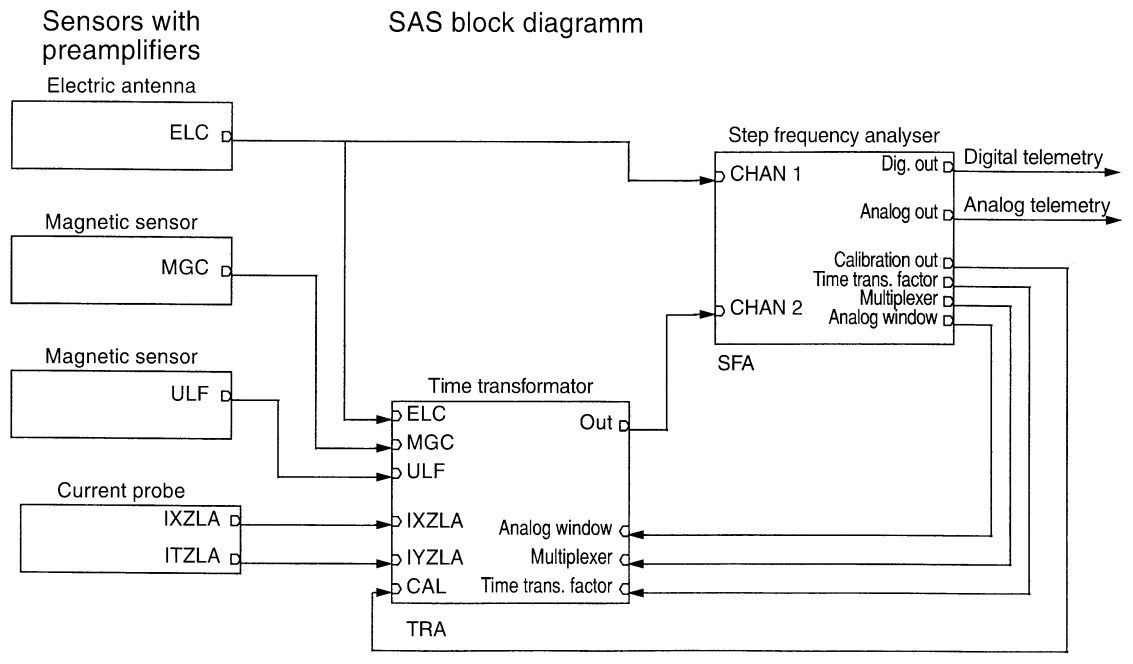

Fig. 1. Block scheme of the SAS experiment

perpendicular to it. The sensitivity of these measurements is $3 \cdot 10^{-5} \mathrm{nT} \mathrm{Hz} z^{-1 / 2}$ at $100 \mathrm{~Hz}$. The output signal of the detectors is processed by the step-frequency spectrum analyser: SAS.

\subsection{Spectrum analyser}

SAS is a two-channel step-frequency spectrum analyser. Each channel can be switched on and off by telecommands. The additional part of SAS is the time transformer SASTRA, a device expanding the possibility of the analysis into the low-frequency band; signals coming in the $32-2000 \mathrm{~Hz}$ band are transformed into 400 $262544 \mathrm{~Hz}$ band and then analysed by SFA. A summary of the technical characteristics of SAS is as follows: frequency range $-32-2000 \mathrm{~Hz}$; frequency resolution $32.7 \mathrm{~Hz}$; dynamic range $->80 \mathrm{~dB}$; time of analysis -20 ms step ${ }^{-1}$; max. data rate $-4 \mathrm{kbits} \mathrm{s}^{-1}$; number of steps max. 64 (linear).

\section{First results}

The number of available measurements from the subsatellite Magion 4 is very limited. There is no memory for the scientific data in the telemetry system on the subsatellite. It means that only the data collected during direct transmission can be transmited to the telemetry station, which is located in Panska Ves in the northern part of the Czech Republic (Triska et al., 1995).

There are other problems with the wave experiment. It consumes a high amount of electric energy and the periods during which SAS was switched on were rather short and rare, particularly for the first two months of operation; indeed the experiment was switched off during the first crossings of the bow shock and magnetopause. The regular measurements by SAS began in October 1996, when the satellite Interball 1 and subsatellite Magion 4 were in the magnetospheric tail. We present results of wave measurements performed in
February-May 1996 in the magnetospheric tail, at the magnetopause and within the polar cusp. The timeintervals of the measurements were selected for the predicted crossings of the interesting structures.

The results presented in this paper are preliminary, and our aim is to use them only as an example of the possibilities of the wave measurements by the SAS instrument on board Magion 4 in different regions of the magnetosphere.

\subsection{The magnetospheric tail}

The first measurements of the plasma waves in the plasma sheet were presented by Scarf et al. (1974) and Gurnet et al. (1976). Their study and many further investigations (Klimov et al.; 1986, Baumjohann et al., 1986; Cattell and Mozer, 1987, Zelenyi and Buchner, 1988, Błęcki, 1995) indicate that intensity of plasma waves in the plasma sheet increases with the geomagnetic activity. The knowledge of the plasma-wave modes and their amplitude is important for the study of the dynamics and microphysics of the processes in the magnetotail, particularly during the development of substorms. A day with high magnetic activity $\left(K_{p}=3\right)$ was 23 February 1996. The Magion4 subsatellite crossed the plasma sheet at the evening flank in a very interesting region not yet well studied; the co-ordinates of the subsatellite in the GSE system during the discussed interval of time were $r=10.5 \mathrm{R}_{\mathrm{E}}$, MLT = 18:57, GMLAT $=3.2^{\circ}$. The perspective view of the spectra taken within the plasma sheet is presented in Fig. 2. The vertical axis represents the spectral density of the magnetic-field fluctuations $\left(B_{x}\right.$-component) in the telemetric units, while the two in the horizontal plane correspond to frequency and the universal time, respectively. Figure 3 shows single spectrum of the magneticfield fluctuations ( $B_{x}$-component) at the moment when the value of the DC magnetic field measured on board the main satellite dropped to $12 \mathrm{nT}$. The characteristic maxima appear at about 200, 400 and $600 \mathrm{~Hz}$, and are likely associated with harmonics of the electron gyro- 


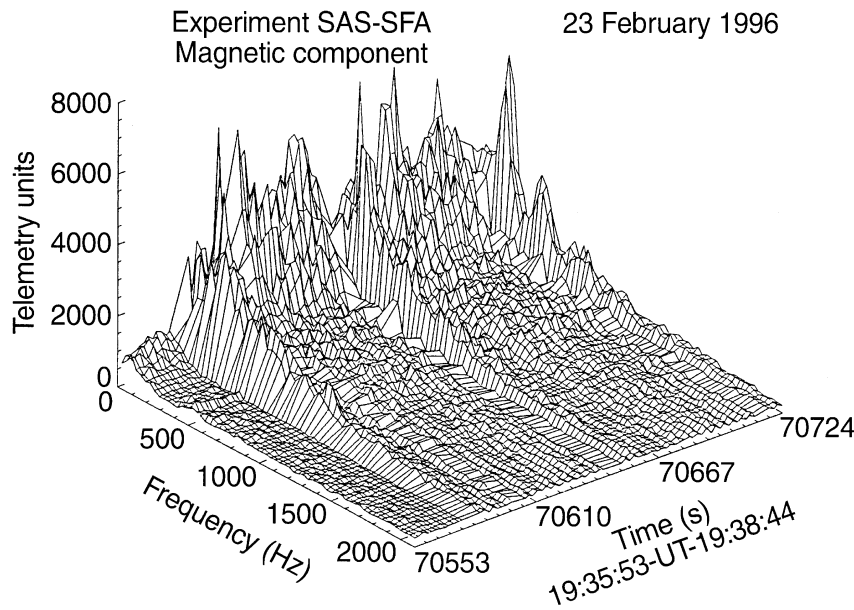

Fig. 2. A panoramic view of the magnetic-field ( $B_{x}$-component) fluctuation spectra in the frequency range $32-2000 \mathrm{~Hz}$ taken within the plasma sheet on 23 February 1996 during disturbed time $\left(K_{p}=3\right)$. Horizontal axes represent frequency and universal time, the vertical spectral density in the telemetric units. The co-ordinates: geocentric distance, magnetic local time and magnetic latitude of the satellite during the time-interval of the measurements are $10.5 \mathrm{R}_{\mathrm{E}}, 18.57,3.3^{\circ}$. The increase in intensity just above 250 and $500 \mathrm{~Hz}$ at 19:37 UT and at the end of the interval of the measurements is associated with harmonics of the electron cyclotron frequency

frequency, which at that moment was about $300 \mathrm{~Hz}$. These modes of plasma waves can be generated by microinstabilities of the anisotropy of the electron temperature or ring distribution of the electrons (Mikhailovsky, 1970). This type of electron distribution function is often observed in the near-Earth plasma sheet.

\subsection{The magnetopause}

Plasma waves are a permanent feature of the magnetopause (Anderson et al., 1982). The characteristic modes occurring at it are the Langmuir plasma waves (Ander-

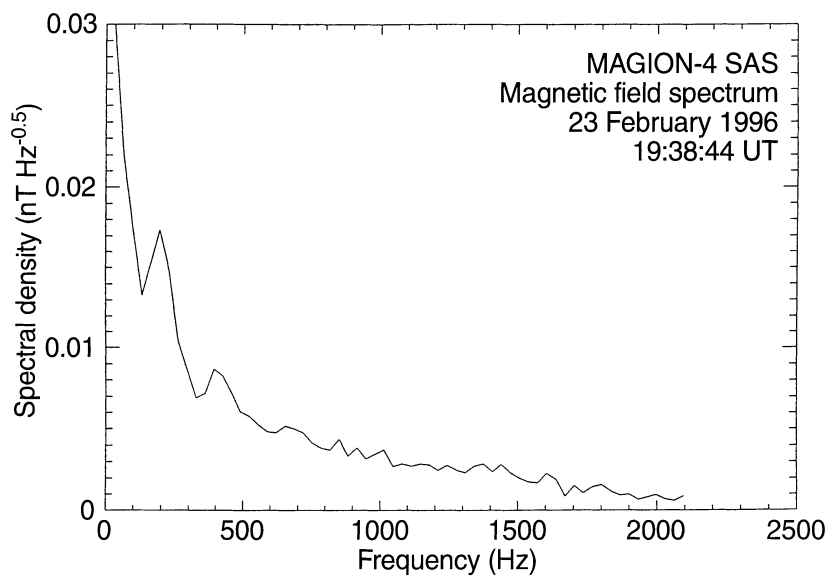

Fig. 3. The spectrum of the magnetic-field ( $B_{x}$-component) fluctuations taken within the plasma sheet. The maxima at 200 and $400 \mathrm{~Hz}$ correspond to the harmonics of electron gyro frequency

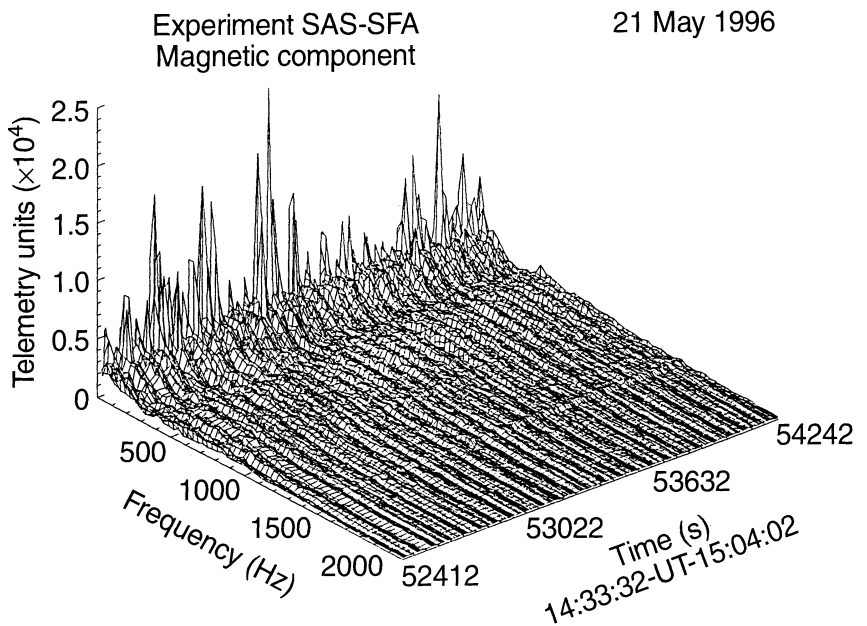

Fig. 4. The same as in Fig. 2 for the magnetopause crossing on 21 May. The sharp enhancement at the lowest frequency can be seen at 14:39, 14:45 and 14:58 UT. The co-ordinates of the satellite were $r=$ $10.4-11.7 \mathrm{R}_{\mathrm{E}}$, Mlat $=62-57^{\circ}, \mathrm{MLT} \approx 12$

son et al., 1982), ion-cyclotron and lower hybrid modes (Belova et al., 1991, Błęcki et al., 1987). The plasma waves are discussed as a main source of the anomalous transport across the magnetopause and anomalous resistivity in the site of reconnection (Treumann et al., 1995).

An example of the wave spectra measured by SAS during the magnetopause crossing on 21 May by the Magion4 subsatellite is presented in Fig. 4. Three regions of enhanced wave activity are seen, first at 14:39 UT, second at 14:45 UT and last at 14:58-15:03 UT, when the final crossing of the magnetopause took place. The most intensive emission appears in the first channel at the frequency $32 \mathrm{~Hz}$ and is likely to be a magnetic component of the lower hybrid mode. The elevated level of the spectral density is seen between 0.5 and $1 \mathrm{kHz}$ in Fig. 5. This frequency range corresponds to the electron-cyclotron mode in the vicinity of the magnetopause. The co-ordinates of the satellite during

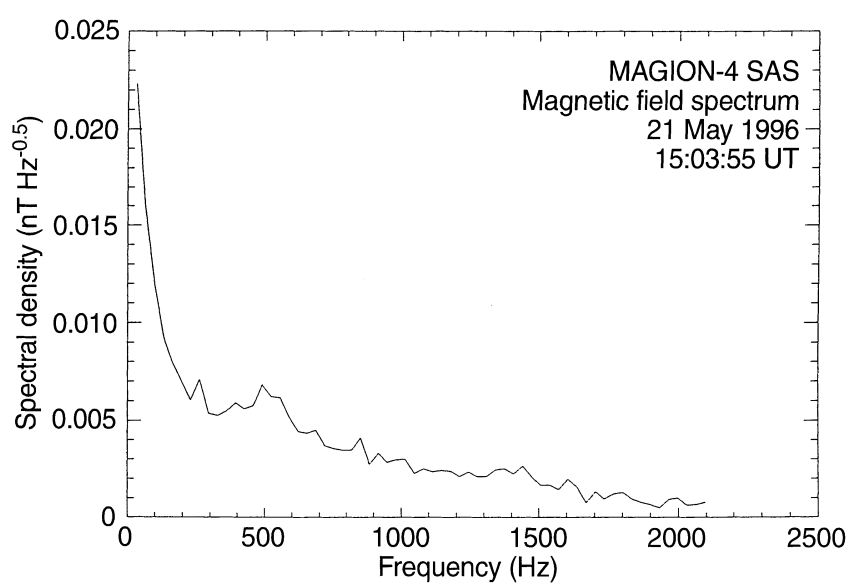

Fig. 5. The spectrum of the magnetic-field ( $B_{x}$-component) fluctuations taken during the magnetopause crossing 


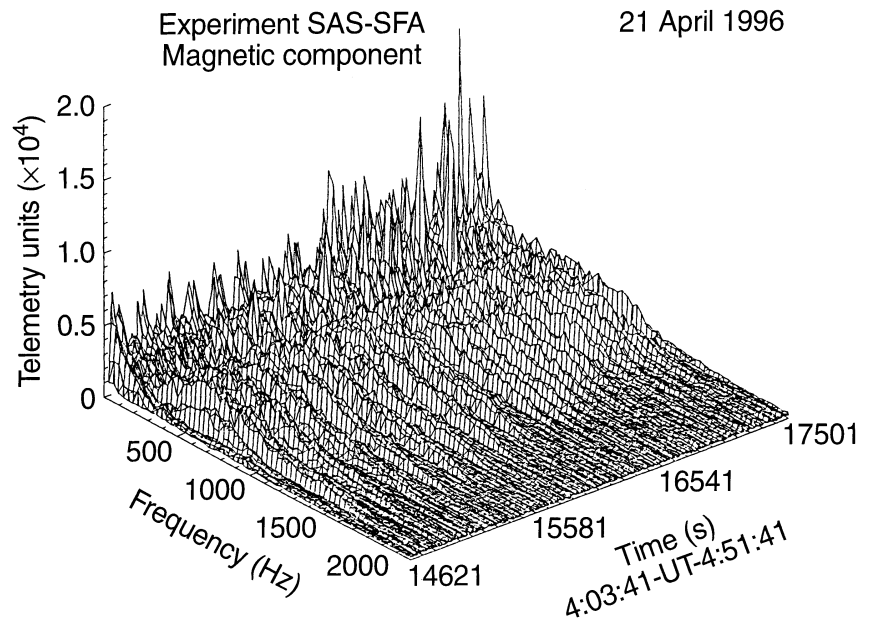

Fig. 6. The same as Fig. 2 during the flight within the polar cusp

the discussed time-interval of the measurements were $r=10.4-11.7 \mathrm{R}_{\mathrm{E}}$, magnetic latitude $62^{\circ}-57^{\circ}$ local magnetic time close to 12 .

\subsection{The polar cusp}

The most turbulent region of the magnetopause-polar cusp is the crucial region of the solar wind-magnetosphere interaction. It is an excellent opportunity to conduct the study of the plasma-wave generation mechanisms and the role of the waves in the processes of particle acceleration and energisation. The modelling of the solar wind flow around the magnetosphere shows that cusp has a very complicated structure of the magnetic field and the plasma flow. There are vortices and turbulent structures with the plasma beams and electromagnetic emissions (Erlandson et al., 1988). Interball 1 flew along the polar cusp on 21 April from 04:05 until 04:45 UT, when the magnetopause was finally crossed. Figure 6 shows the perspective view of the magnetic-field fluctuation spectra ( $B_{x}$-component) in the frequency range $32-2000 \mathrm{~Hz}$ obtained within the

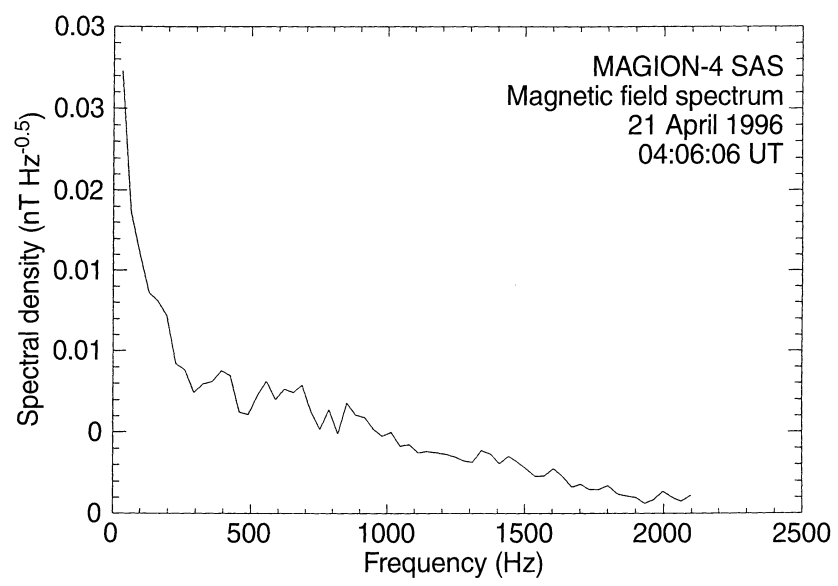

Fig. 7. The spectrum of the magnetic-field ( $\mathrm{B}_{x}$-component) fluctuations at the leading edge of the cusp

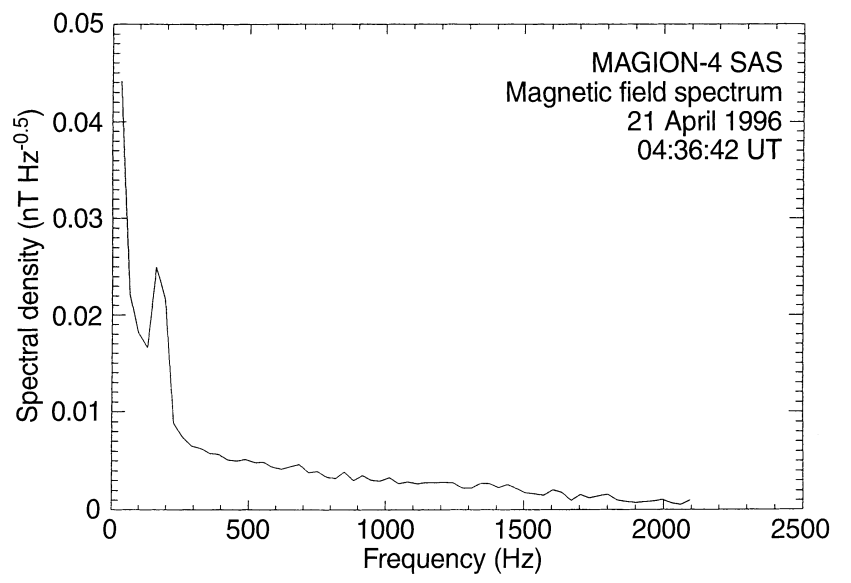

Fig. 8. The same as in Fig. 7 but at the edge of the entry layer or plasma mantle

cusp. It can be seen that the elevated level of the emissions appears in the broad band of the frequency from $32 \mathrm{~Hz}$ up to $1500 \mathrm{~Hz}$. The enhancement of the intensity in the lower part of spectra at 04:40UT corresponds to the approach to the magnetopause and encounter with entry layer (EL) or plasma mantle (PM) seen by the Czech plasma instrument MPS (Principal Investigator of this instrument is Z.Nemecek). The characteristic spectra taken at the leading edge of the polar cusp and at the entrance into EL or PM are shown in Figs. 7 and 8, respectively. The polar cusp is a region where the magnetosheath plasma is compressed and accelerated. The pick of the intensity seen at the frequency $150 \mathrm{~Hz}$ (Fig. 8) can be associated with the electromagnetic emission called "lion roar" characteristic for the magnetosheath (Anderson et al., 1982).

\section{Conclusions}

In spite of the technical difficulties and some limitations, the wave experiment SAS gives interesting data on the magnetic-field fluctuation spectra in the frequency range $32-2000 \mathrm{~Hz}$ in different regions of the magnetosphere. Some of the magnetopause and bow-shock crossings were registered, two encounters with the polar cusp and many auroral field-line crossings until May 1996. The results presented here are only preliminary and need further, more complex, physical interpretation together with plasma and magnetic data.

Acknowledgements This work has been supported by grant KBN 2 P03C 00908.

Topical Editor K.-H. Glaßmeier thanks two referees for their help in evaluating this paper.

\section{References}

Anderson, R. R., C. C. Harvey, M. M. Hoppe, B. T. Tsurutani, B. T. Eastman, and J. Etcheto, Plasma waves near the magnetopause, J.Geophys. Res., 87, 2087, 1982.

Baumjohann, W., R. A. Treumann, and J. LaBelle, Average electric wave spectra in the plasma sheet: dependence on ion density and ion beta, J.Geophys.Res., 95, 3811-3817, 1986. 
Belova, E., V., J. Błęcki, M. Denis, L. M. Zelenyi, and S. P. Savin, Ion - cyclotron wave generation at the magnetopause, Sov. Plasma Phys., 17, 952, 1991.

Błęcki J., ULF/ELF plasma waves in the magnetosperic tail as observed by Prognoz-8, ESA SP-371, 247, 1995.

Błęcki, J., K. Kossacki, S. J. Klimov, M. N. Nozdrachev, A. N. Omelchenko, S. P. Savin, and A. J. Sokolov, ELF/ULF plasma waves observed on Prognoz-8 near the magnetopause, Art. Sat. Space Physics, 22, (4), 5, 1987.

Cattel, C. A., and F. S. Moser, Substorm-associated lower hybrid waves in the plasma sheet observed by ISEE 1, in Magnetotail Physics, ed. A.T.Y.Lui, Johns Hopkins University Press, Baltimore, London, p 119, 1987.

Erlandson, R. E., L. J. Zanetti, T. A. Potemra, M. Andre, and L. Matson, Observations of electromagnetic ion cyclotron waves and hot plasma in the Polar Cusp, Geophys. Res. Lett., 15, 421, 1988.

Gurnet, D. A., L. A. Frank, R. P. Lepping, Plasma waves in the distant magnetotail, J.Geophys.Res., 81, 6059, 1976.

Klimov, S. I., S. A. Romanov, M. N. Nozdrachev, S. P. Savin, A. J. Sokolov, L. M. Zelenyi, J. Błęcki, K. Kossacki, P. Oberc, B. Popielawska, J. Buchner, and B. Nikutowski, Comparative study of the plasma wave activity in the plasma sheet boundary layer and near-Earth plasma sheet, Adv.Space Res., 6, 153,1986.

Klimov, S. I., S. Romanov, E. Amata, J. Błęcki, J. Buchner, J. Juchniewicz, J. Rustenbach, P. Triska, L. J. C. Woolliscroft, S. Savin, Yu. Afanasyev, U. deAngelis, U. Auster, G. Bellucci, A. Best, F. Farnik, V. Formisano, P. Gaugh, R. Grard, V. Grushin, G. Haerendel, V. Korepanov, H. Lehmann, B. Nikutowski, M. Nozdrachev, S. Orsini, M. Parrot, A. Petrukovich, J. L. Rauch, K. Sauer, S. Skalsky, J. Słomiński, J. G. Trotignon,
J. Vojta, and R. Wronowski, ASPI experiment measurements of fields and waves onboard the Interball-Tail mission, in INTERBALL Mission and Payload, Russian Space Agency, IKI, CNES, 120-152, 1995.

Klimov, S. I., S. Romanov, E. Amata, J. Błęcki, J. Buchner, J. Juchniewicz, J. Rustenbach, P. Triska, L. J. C. Woolliscroft, S. Savin, Yu. Afanasyev, U. deAngelis, U. Auster, G. Bellucci, A. Best, F. Farnik, V. Formisano, P. Gaugh, R. Grard, V. Grushin, G. Haerendel, V. Korepanov, H. Lehmann, B. Nikutowski, M. Nozdrachev, S. Orsini, M. Parrot, A. Petrukovich, J. L. Rauch, K. Sauer, S. Skalsky, J. Słomiñski, J. G. Trotignon, J. Vojta, and R. Wronowski, ASPI experiment measurements of fields and waves onboard the Interball-Tail mission, Ann. Geophysicae, this volume, 1997

Mikhailovsky, T., Theory of Plasma Instabilities, (In Russian), Atomizdat, Mascow 1970.

Scarf, F.L., L. A. Frank, K. L. Anderson, and R. P. Lepping, Plasma wave turbulence at the distant crossings of the plasma sheet boundaries and neutral sheet, Geophys. Res. Lett., 1, 109, 1974.

Shawhan, S. D., Magnetospheric Plasma Waves, in Solar System Plasma Physics vol. III, eds C.F.Kennel, L.J.Lanzerotti, and E.N.Parker, North-Holland, Amsterdam, A211 1979.

Treumann, R.A., J. LaBelle, and T. M. Bauer, Diffusion processes: an observational perspective, in Physics of the Magnetopause, Eds. P.Song, B.U.O.Sonnerup, and M.F.Thomsen, American Geophysical Union, Washington D.C., p. 3311995.

Triska, P., Vojta J., and Yu. Agafonov, The INTERBALL subsatellites S2-A and S2-T, in INTERBALL Mission and Payload, Russian Space Agency, IKI, CNES, p. 100, 1995.

Zelenyi, L. M., and J. Buchner., Wave Processes in the magnetotail (in Russian), Issl. Kosm. Prostranstva, VINITI, 28, 3, 1988. 